\title{
A Design of Active Power Filter for Superconducting Nanowire Single-Photon Detectors
}

\author{
Wang En-qiang ${ }^{1,}$, , Zhang La-bao ${ }^{2}$, Zhang Xiao-ying ${ }^{1}$ \\ ${ }^{1}$ Department of Electrical Engineering and Information Engineering, Lanzhou University of Technology, Lanzhou, China \\ ${ }^{2}$ Department of Electronic Science and Engineering, Nanjing University, Nanjing, China
}

Email address:

enqiang123@126.com (Wang En-qiang), Lzhang@nju.edu.cn (Zhang La-bao),245659219@qq.com (Zhang Xiao-ying)

\section{To cite this article:}

Wang En-qiang, Zhang La-bao, Zhang Xiao-ying. A Design of Active Power Filter for Superconducting Nanowire Single-Photon Detectors. Journal of Electrical and Electronic Engineering. Vol. 3, No. 5, 2015, pp. 170-176. doi: 10.11648/j.jeee.20150305.22

\begin{abstract}
The basic structure and working principles of superconducting nanowire single-photon detectors (SNSPD) are described in this paper. The special requirements for power quality of the system are introduced in this paper. Related experiments are designed to measure the power quality of the system. The paper designs a new type of single-phase active power filter(APF). By applying selective harmonic elimination technique(SHE), the APF can compensate the reactive component and high harmonics of line current without detecting the reactive current and harmonic current of the load. A switch angle's solution method of optical SHEPWM is presented. PI controller of the APF is also designed. According to the data we got, the APF we designed can attain the goal of eliminating harmonics effectively.
\end{abstract}

Keywords: Superconducting Nanowire Single-Photon Detectors, SHE PWM, Active Power Filter

\section{面向超导纳米线单光子探测系统的有源电力滤波器设计}

\author{
王恩强 ${ }^{1, *}$, 张蜡宝 ${ }^{2}$, 张晓英 ${ }^{1}$ \\ ${ }^{1}$ 电气工程与信息工程学院, 兰州理工大学, 兰州, 中国 \\ ${ }^{2}$ 电子科学与工程学院, 南京大学, 南京, 中国
}

\section{邮箱}

enqiang123@126. com(王恩强), Lzhang@nju. edu.cn(张蜡宝)，245659219@qq. com(张晓英）

摘要: 本文阐述了超导纳米线单光子器探测系统的基本结构和工作原理, 介绍了系统对于电能质量的特殊要求。设计 了相关实验对实验室的电能质量进行测量分析。设计了一种新型的单相有源电力滤波器 (APF), 应用特定消谐技术, 不 需实时检测负载的无功电流和谐波电流, 即可实现同时补偿负载无功及高次谐波电流的需求。给出了一种优化特定谐 波消除PWM技术开关角的求解方法。设计了单相有源电力滤波器PI控制器。通过前后电能质量指标的对比, 可知该滤波 器达到了消除和抑制谐波的目的。

关键词：超导纳米线单光子探测系统，特定谐波消除PWM，有源电力滤波器

\section{1. 引言}

超导纳米线单光子探测器 (SNSPD) 是一种最新的单 光子检测技术, 具有暗计数低、探测速度快、响应频谱宽 和效率高等特点, 是当前单光子探测技术研究领域中的研
究热点。由于实验室供电微弱的谐波都会对单光子探测器 的探测性能产生很大的影响, 因此我们需要通过一些措施 改善实验室供电的电能质量, 即减少谐波对于超导纳米线 单光子探测器检测系统的干扰。 
为了减小谐波问题给用户带来的危害, 各国研究人员 对谐波和无功的特性进行了深入的研究, 并提出了相应的 补偿方法 $\left[1^{\sim} 7\right]$ 。文献 $[8]$ 介绍了 $i_{p}-i_{q}$ 法和网侧电流法两种 谐波检测方法的有源电力滤波器, 对比传统的有源电力滤 波器 $i_{p}-i_{q}$ 谐波检测方法的复杂性, 提出了网侧电流检测 法, 此种方法不仅省去了传统有源电力滤波器的谐波检测 单元，而且更为简单实用。文献 [9] 针对并联型有源电力 滤波器提出一种基于内模控制原理的PI控制策略, 利用该 方法对并联型有源电力滤波器系统电流内环与直流侧电 压外环PI控制器进行设计, 以提高系统的稳定性和动态性 能, 简化PI控制器设计过程。文献 [10]提出一种直接电流 控制和倍频载波相移SPWM相结合的级联型APF控制技术， 具有易于实现和补偿效果好等优点。文献 [11]中阐述了如 何在数字信号处理器中分别用线性插值法和牛顿迭代法 实现特定谐波消除脉宽调制技术开关角的计算。总之, 一 般是通过装设谐波和无功补偿装置来补偿谐波, 并提高功 率因数, 这就是通常所说的电力滤波器。电力滤波器一般 分为无源电力滤波器和有源电力滤波器, 其中无源电力滤 波器是目前使用最为广泛和成熟的谐波抑制手段, 但是无 源电力滤波器存在一些固有的缺点: 可能导致谐振现象、

系统的阻抗严重地影响谐波特性、无法对变化的谐波进行 实时补偿等 [12]。为了解决无源滤波器的问题, 人们提出 了有源电力滤波器的方案。目前的研究多是对三相系统进 行补偿, 对于单相系统研究的很少, 所提出的单相APF 的 方法都有一定的缺陷, 基于自适应干扰对消原理的有源滤 波器采用开环控制, 稳定性比较差 [13]; 单周控制 [14] 需要快速复位的积分电路, 开关误差校正能力有限, 系统 存在稳态误差, 在负载电流快速发生变化时无法准确复位, 导致电源电流有突变; 80 年代末, 加拿大Phoivos D. Ziogas等人提出的三相电压源型APF [15], 能够用于无 功和谐波补偿。既可以应用于三相系统中, 又可以应用于 单相系统中, 它不需要实时检测负载电流的无功和谐波分 量, 只通过调节逆变器输出电压的幅值和相位就可以实现 对电网进线端电流的谐波和无功补偿, 很好地解决了电流 跟踪型APF的缺点 [16]。本文将牛顿迭代算法应用到特定 消谐技术中, 求解速度快, 根据前后数据的对比, 证明了 该种方法的可行性。

\section{2. 超导纳米线单光子探测系统基本结构与工作 原理}

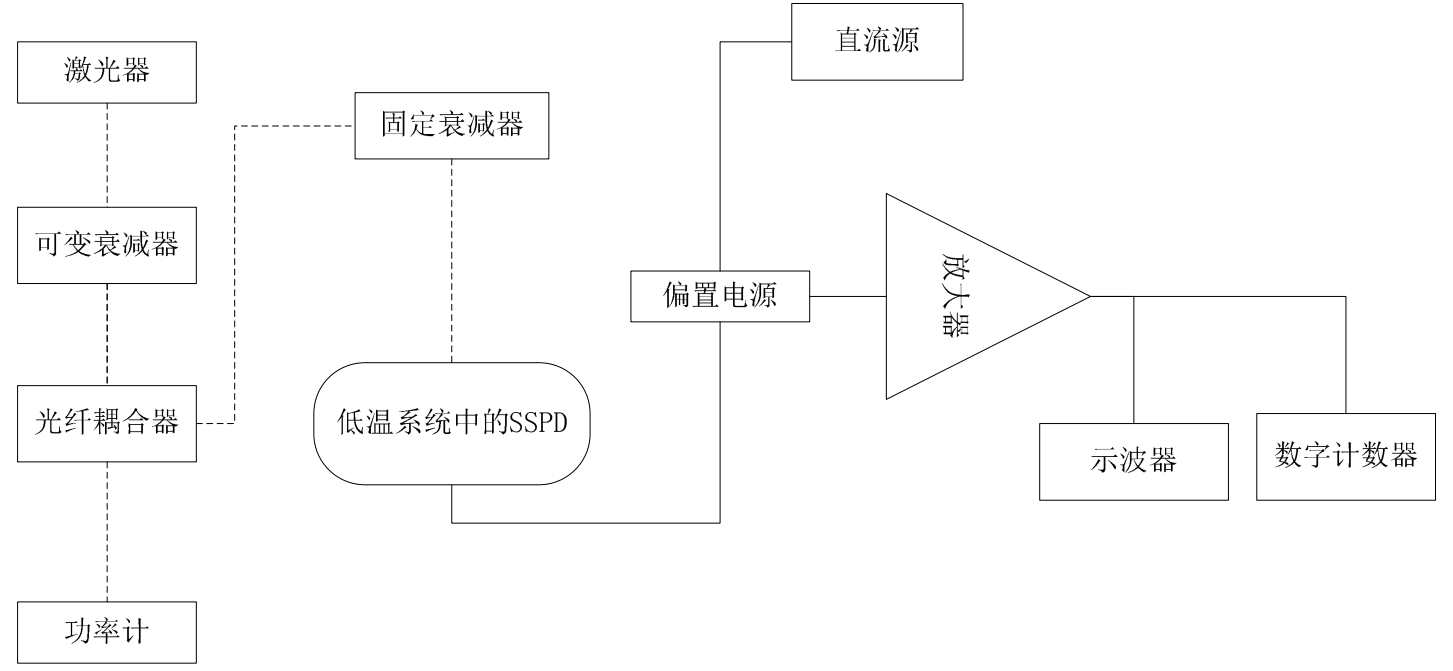

图1 超导纳米线单光子探测系统的基本结构示意图。

如图1所示, 超导纳米线单光子探测系统包括: 光路 系统 (图中用虚线连接), 低温系统和测量电路（图中用 实线连接）。光路系统为单光子探测系统提供光子源, 本 系统采用多个衰减器将入射激光脉冲衰减至平均光子数 小于 0.1 光子的强度, 在此强度下, 超过 $95 \%$ 的光子为单光 子。衰减器起到了模拟单光子源的作用。激光脉冲通过可 调衰减器之后分别输入到固定衰减器和光功率计, 通过固 定衰减器进一步衰减的信号通过光纤传输到低温系统并 耦合到SNSPD探测区域。这里固定衰减器的衰减倍率可以 提前测定, 因此, 通过光功率计可以估算入射到SNSPD上 的光子数。低温系统是小型的 GM制冷机, 在温度为 $2.3 \mathrm{k}$ 时, 纳米线处于超导状态。测量电路是利用同轴电缆将 SNSPD连接到偏置电源, 再连接到放大器, 经过放大器的 放大后, 将信号传送给示波器和计数器。计数器用来显示 光子数, 示波器横轴为时间, 坚轴为电压。由于光纤处于
超导状态, 当光子打到上面的时, 局部温度升高超导态被 破坏, 电阻升高, 此时产生一个电压值, 所以每一个光子 对应一个电压, 从示波器的坚轴上的电压值能读出光子个 数。由此可得到单光子探测器的探测速度。

如果电网的电能质量较差或谐波含量较高可能会使 测量系统暗计数提高, 从而无法正确测量单光子探测器的 性能。所以, 实验室电能质量的改善就显得非常重要。

\section{3. 单相有源滤波器的设计}

\section{1. 超导纳米线单光子探测系统供电电能质量检测与分 析}

超导纳米线单光子探测系统采用单相供电, 所以选择 Fluke 43B型电能质量分析仪对系统进行电能质量的检测。 
该实验从2015年9月30日到2015年10月7日连续8天每天定 时对系统的电能质量进行测量。经连续测量, 电压和电流
的波形都是标准的正弦波。以2015年9月30日上午9点 40 分测量结果为例, 其电压、电流波形如图2所示。

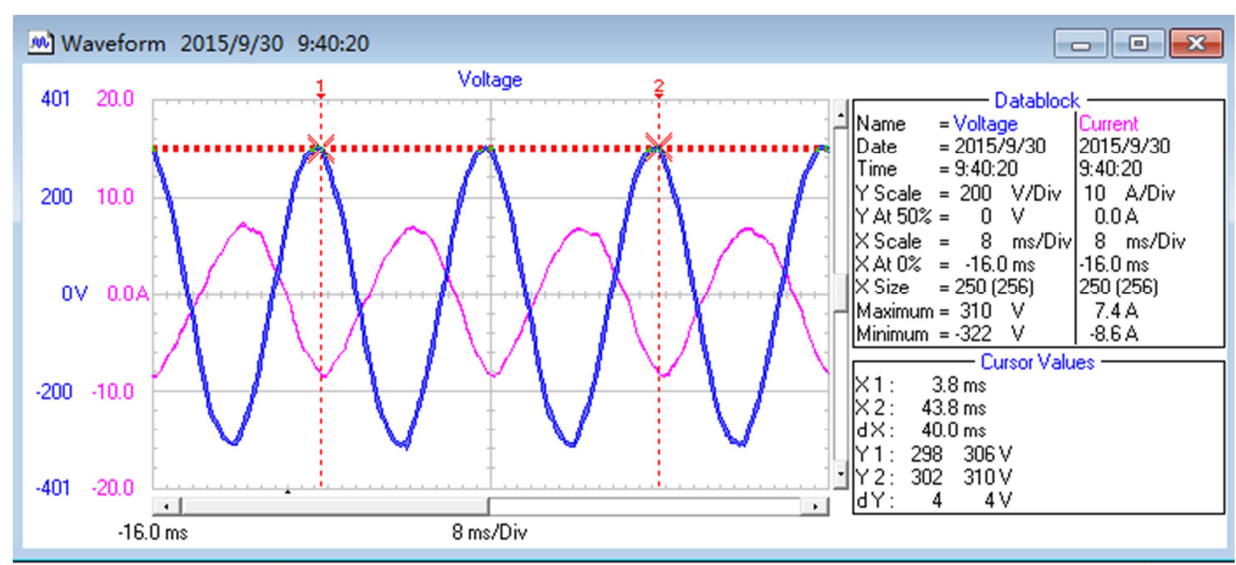

图2 2015年9月30日9:40时的电压与电流波形图。

每天的谐波总畸变率 (THD) 如图3所示。

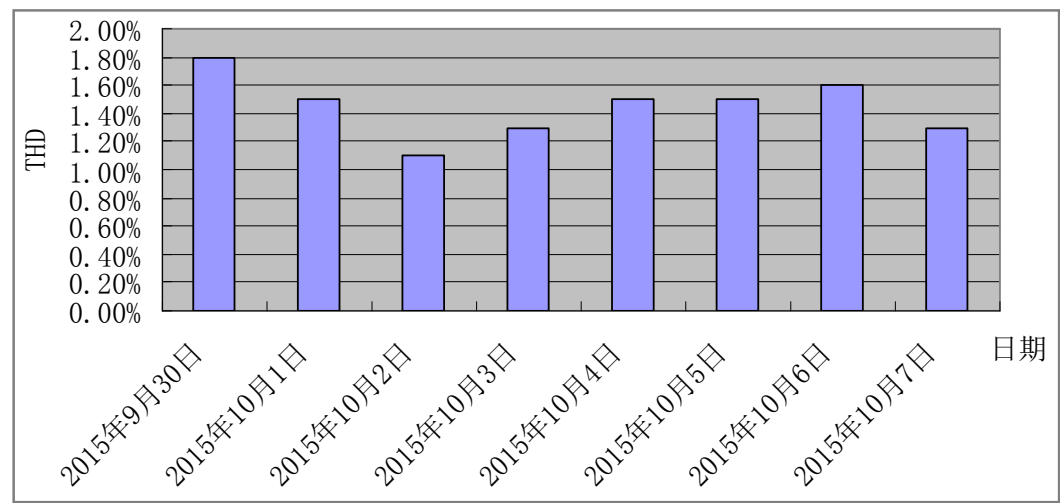

图3 2015年9月30日至2015年10月7日系统供电的谐波总畸变率。

以2015年9月30日9: 40测量情况为例，所测得的谐波含量如图4所示。

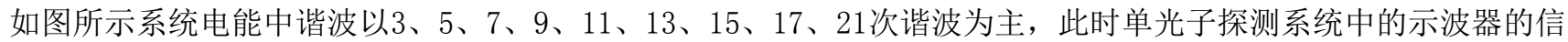
号噪声较大, 在这种环境下进行测量, 暗计数会变高, 测量不准确。

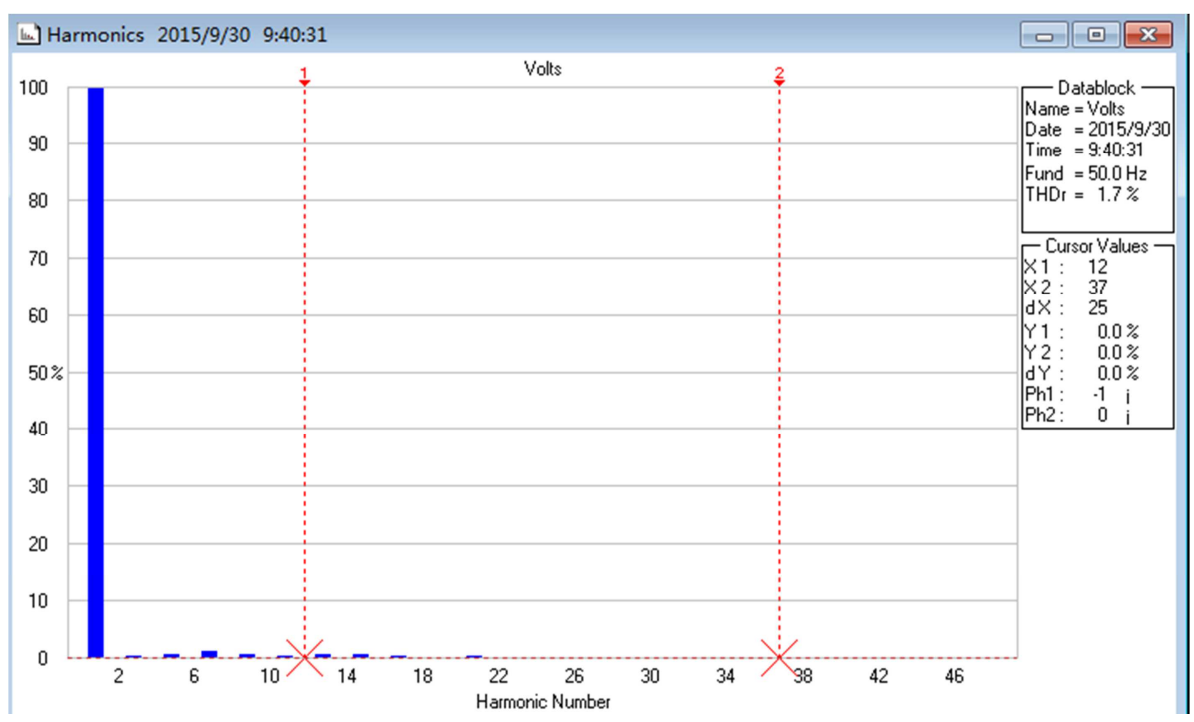

图4 2015年9月30日9: 40时系统供电的谐波含量。 


\section{2. 单项有源电力滤波器的系统组成和工作原理}

本文设计的 $\mathrm{APF}$ 由消除特定次谐波的PWM电压型逆变 器构成, 它强迫逆变器输出端电压为正弦的电压源。由于 逆变器交流侧电压输出仅由直流侧的电压以及开关所采 取的调制方式确定, 当逆变器直流侧为一恒定电压源且开 关控制采用特定消谐PWM技术时, 其交流侧输出电压主要
包括基波以及PWM调制频率及其倍频附近的高次谐波。因 此, 将逆变器并联在负载侧, 如能保证逆变器直流电容的 电压稳定, 则负载的输入电压将被强制为逆变器的输出电 压。当采用适当的调制方式使逆变器的输出为正弦波时, 即可保证用户的电压质量, 而且可抑制非线性负荷产生的 谐波注入电网。

单相有源滤波器结构如图5所示。

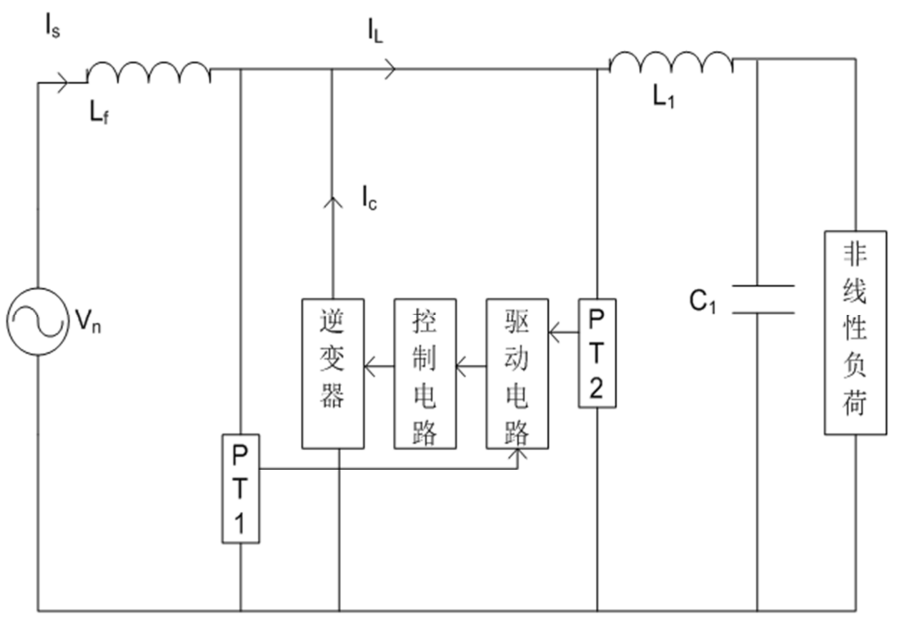

图5 单相有源电力滤波器结构示意图。

如图5所示: $I_{s}$ 为电源电流, $I_{L}$ 为负载侧电流, $I_{C}$ 为 逆变器输出电流, 电网电压 $V_{n}$ 通过补偿电感 $L_{f}$ 与 $\mathrm{H}$ 桥逆 变器并联, 电压互感器PT1、PT2 分别检测控制回路所需 的电网电压, 逆变器输出电压。为了减小负载进线电压的 畸变程度, 在逆变器输出端和负载输入端连接了由 $L_{1}$ 作 为高次谐波滤波器。

图6为电力有源滤波器单相等值电路, $V_{i}$ 为逆变器输 出电压, 具体实现原理如下:

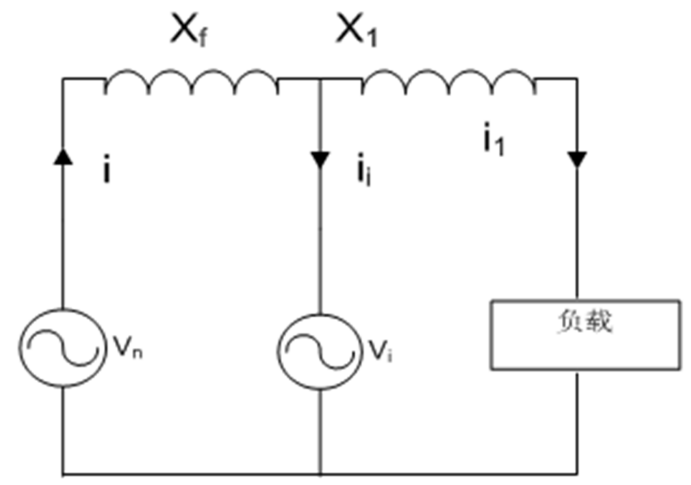

图6 有源电力滤波器单相等值电路。

1) 电压补偿通过维持 $V_{i}$ 恒定实现;

2) 逆变器输出电压 $V_{i}$ 滞后于交流电网电压 $V_{n}$ 的移 相角为 $\delta$, 输入电流 $\mathrm{i}$ 滞后于系统侧一阶低通滤波器的电 压大约 $90^{\circ}$;
3) 由交流电网向负载和补偿器传递的有功功率可由 式 (1) 决定:

$$
P=\frac{V_{n} V_{i}}{X_{f}} \sin \delta
$$

流入逆变器的有功功率为电网输出有功功率 $P$ 与负载 消耗的有功功率之间的差额, 由于稳态运行时滤波器的损 耗基本不变, 因此改变移相角 $\delta$ 可以调节负载消耗的有功 功率, 从而迫使负载电压改变。因为电力有源滤波器运行 于一种自足方式, 即逆变器直流侧没有额外的功率输入, 因此逆变器输出端电压 $V_{i}$ 的幅值可以通过调节相角差 $\delta$ 来实现。在系统进入稳态运行时, PWM电压型逆变器仅 从电网吸收少量的有功功率来补偿直流电容与开关器件 的损耗, 以维持 $V_{i}$ 的幅值恒定。

1）负荷动态调节

当电网电压低于额定电压时, 为了维持负载电压恒定, 必须保证 $V_{i}$ 的幅值恒定, 此时应增大 $V_{i}$ 角, 由式 (1) 可知, $\delta$ 角增大将使得电网向补偿器与负载提供的有功功率增 加, 由于逆变器所消耗的有功功率基本不变且很小, 实际 分析时可认为近似为零, 因此负载消耗的有功功率将增加, 如果认为负载电阻不变, 则负载电压将上升, 此时逆变器 的直流侧电容被充电, 直至直流电容电压 $V_{d c}$ 增加到需要 的值。同理, 当电网电压过高时, 将产生相反的变化来维 持直流电容电压的恒定。

2）负荷无功功率调节 
当控制逆变器输出端电压 $V_{i}$ 和系统侧电压 $V_{n}$ 之间的 相角差 $\delta$ 较大时, 系统侧注入负荷侧的有功功率 $P$ 增加, 在负荷阻抗固定的情况下将迫使负荷端电压 (逆变器输出 端电压) 增加, 以使注入系统的有功功率与负荷吸收的有 功功率相平衡, 此时, 补偿器向系统注入感性无功功率, 功率因数为超前的功率因数。同理, 控制逆变器输出端电 压 $V_{i}$ 和系统侧电压 $V_{n}$ 之间的相角差 $\boldsymbol{\delta}$ 较小时, 补偿器向
系统注入容性无功功率, 功率因数为滞后的功率因数, 从 而补偿器起到调节负荷功率因数的作用。

\section{3. 优化特定消谐PWM技术 (SHEPWM)}

1973年，美国著名电力电子学专家Richard G. Hoft 提出了特定消谐PWM技术 ${ }^{[15]}$, 此技术可以有效地消除低次 谐波。和其它调制技术相比, 特定消谐技术有许多优势, 开关频率低、输出电压、电流的质量较高 ${ }^{[16]}$ 。双极性SHEPWM 的原理如图7与式 (2), 式 (3)，式（4）所示:

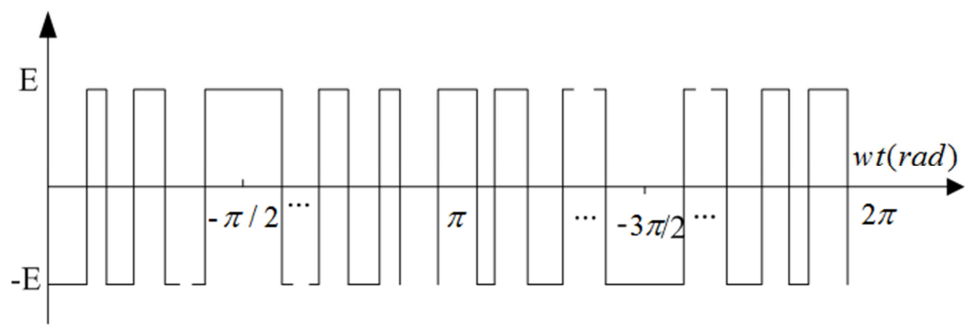

图7 双极性SHEPWM输出电压波形。

$$
\begin{aligned}
& U(t)=\sum_{n=1}^{\infty}\left[a_{n} \sin (n \omega t)+b_{n} \cos (n \omega t)\right] \\
& a_{n}=\frac{1}{\pi} \int_{0}^{2 \pi} U(t) \sin (n \omega t) d(\omega t) \\
& b_{n}=\frac{1}{\pi} \int_{0}^{2 \pi} U(t) \cos (n \omega t) d(\omega t)
\end{aligned}
$$

由于图7中输出电压波形 $U(t)$ 为奇函数, 又是奇谐函 数, 即 $U(t)$ 在 $[0, \pi]$ 区间以 $\pi / 2$ 为轴对称, 在 $[0,2 \pi]$ 区 间以 $\pi$ 点对点对称 [17], 因此使得傅里叶分解式中的直流 分量、余弦分量和偶次正弦分量系数为零, 其傅里叶分解 系数可简化为式 (5) :

$$
\left\{\begin{array}{l}
b_{n}=0, n=1,2,3, \cdots \\
a_{n}=0, n=2,4,6, \cdots \\
a_{n}=\frac{4 E}{n \pi}\left[-1-2 \sum_{k=1}^{N}(-1)^{k} \cos \left(n \alpha_{k}\right)\right], n=1,3,5 \cdots
\end{array}\right.
$$

初值选取是影响算法收敛性的一个关键因素。单相双 极性SHEPWM的初值给定公式见式（6）:

$$
\left\{\begin{array}{l}
\alpha_{1}=90 / N \\
\alpha_{i}=i \times \alpha_{1}, i=2,3 \cdots, N \\
N \leq 21
\end{array}\right.
$$

\section{4. PI调节器设计}

文中实现对相角差 $\delta$ 的控制主要是通过PI调节实现 的, 交流侧输入电压和逆变器输出电压之差作为PI控制器 的输入量, 同时交流侧电压输入锯齿波发生器产生同步的 锯齿波信号, PI控制器的输入量与锯齿波信号通过比较器
比较后, 产生随系统电压和逆变器输出电压之差成线性变 化的可移相方波信号, 此方波信号通过触发器 $\rightarrow$ 计数器 $\rightarrow$ 存储单元 $\rightarrow$ 驱动电路控制主桥路的IGBT输出从而实现 $\delta$ 角的调节改变APF 输出端无功功率的大小和极性。

PI调节器的方程可描述为式 (7) :

$$
\delta(t)=K_{p v} e_{v}+K_{i v} \int_{0}^{t} e_{v} d t
$$

$K_{p v}$ 与 $K_{i v}$ 分别为 $\delta$ 相移控制环节中的PI调节器的 比例和积分系数, $e_{v}$ 为误差电压, $V_{r e f}$ 为电容参考电压。 有式（8）：

$$
e_{v}=V_{r e f}-V_{d c}
$$

通过调节 $K_{i v}$ 提高系统的无差度，进而提高了系统的 稳态性能。当负载稳定时, 系统有好的稳定性; 当负载变 化时, 系统又能及时跟踪负载变化。增大 $K_{p v}$ 可以提高系 统的阻尼程度, 减小系统稳态误差。

本文中 $K_{p v}$ 取值为 $1, K_{i v}$ 取 0.02 。

\section{5. 数据对比}

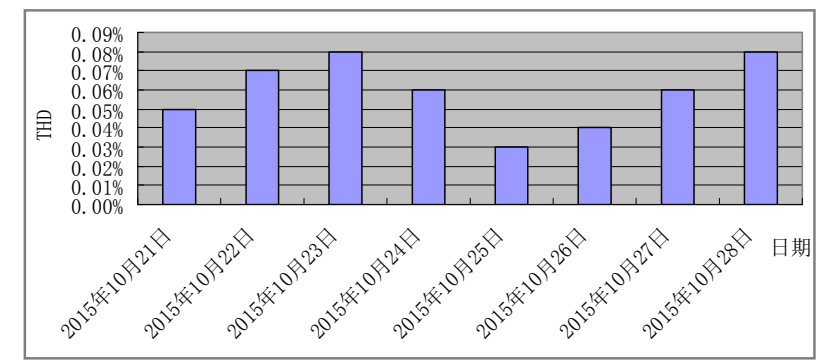

图8 2015年10月21日至2015年10月28日系统供电的谐波总畸变率。 
将经过上述设计的单相有源电力滤波器投入系统使 用后，以2015年10月21日到2015年10月28日为周期，每天 定时对系统电能质量进行测量, 谐波总畸变率如图8所示:

以10月25日为例, 14 点34时谐波含量如图9所示:
通过前后数据的对比可知, 在单相有源电力滤波器投 入使用后, 谐波总畸变率有了大幅下降，3、5、7、9、11、 13、15次谐波被基本滤除，并且示波器的噪声干扰也得到 了很大改善。

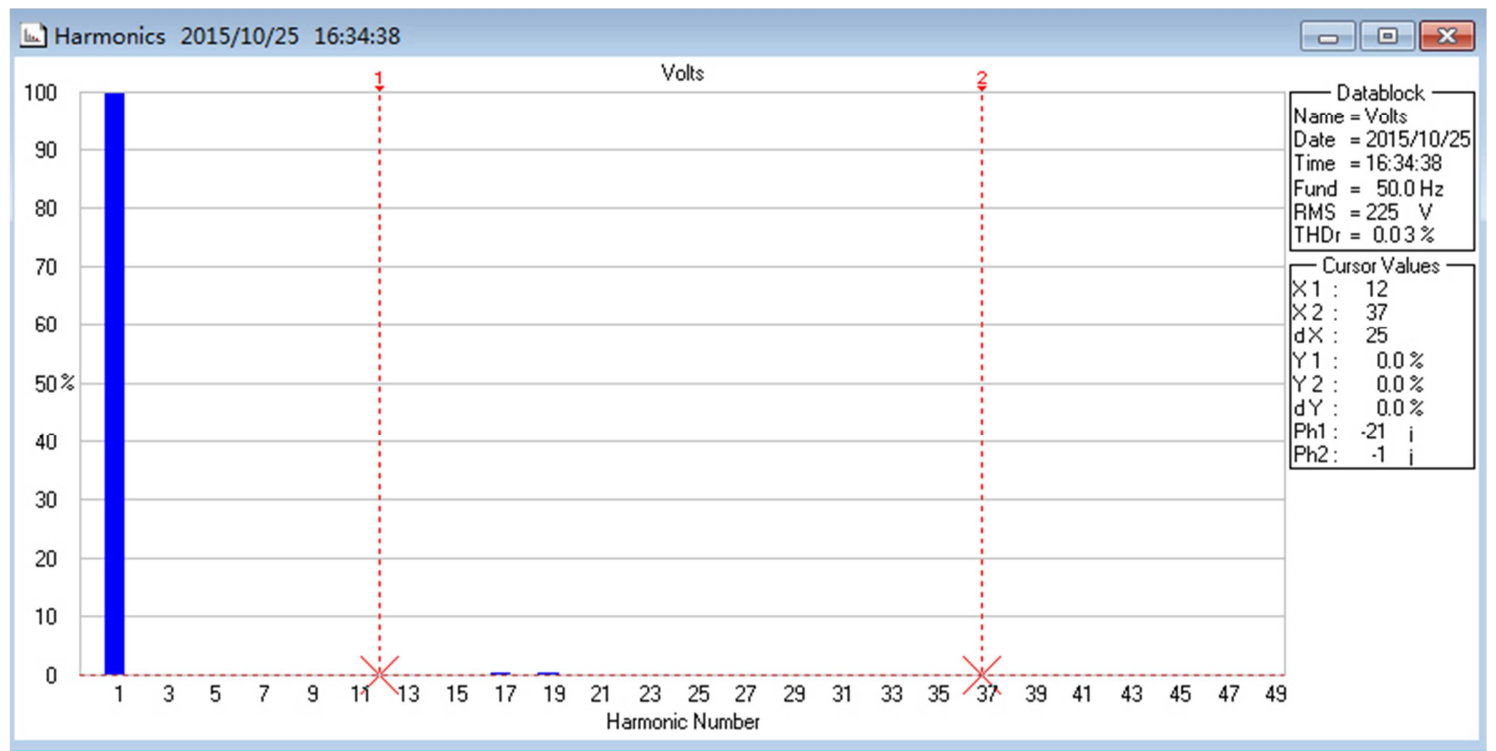

图9 10月25日14点34时的谐波含量。

\section{4. 结论}

本文设计的单相有源滤波器采用了特定消谐技术, 具 有响应速度快，谐波含量小等优点。本文实现的方法强制 逆变器的输出电压为正弦波电压, 因此在某些场合还可以 作为稳定负荷电压使用, 它可以推广应用于电力系统、发 电厂、变电所, 特别适用于电弧炉、牵引变电所和需要抑 制大容量非线性负荷所产生谐波的场所。结果表明, APF 能有效地消除和抑制谐波, 并能灵活地调节无功功率, 具 有广阔的应用前景。

\section{致谢}

本文为国家重大科研仪器设备研究专项《超导纳米线 单光子探测器》的阶段性成果之一。本文是在张蜡宝副教 授和张晓英副教授的精心设计和悉心指导下完成的。导师 渊博的知识、严谨的治学态度、开阔的思路、敏锐的洞察 力、忘我的敬业精神和对学生的高度负责给我留下了深刻 的印象, 这将鞭策和指导学生完成以后的各项工作。同时, 导师的正直、大度和为人处事作风使学生深受裨益, 也将 对学生以后的生活和工作带来深远的影响。在此向导师表 示衷心的感谢和诚挚的敬意。

\section{参考文献}

[1] 王兆安, 杨军, 刘进军. 谐波抑制和无功功率补偿 $[\mathrm{M}]$. 北京: 机械工业出版社, 2002。
[2] 王卫勤, 刘汉奎, 徐殿国, 等. 电压跟踪型单相电网有源 滤波器补偿特性的研究 $[\mathrm{J}]$. 中国电机工程学报, 1997, $19(7)$ : 72-76。

[3] 王建元, 俞红祥, 王琦. 基于DSP新型PWM三相逆变器的研 究 $[J]$. 电力系统及其自动化学报, 2003, 15 (4)：38-41。

[4] Moran L, Ziogas P D, Joos G. A Solid-State High Performance Reactive-Power Compensator [J]. IEEE Trans on Industry Applications, 1993, 29 (5) : 969-978。

[5] 杨君, 王兆安. 三相电路谐波电流两种检测方法的对比研 究 $[J]$. 电工技术学报, $1995,10(2): 43-48$ 。

[6] 杨君, 王兆安, 邱关源. 单相电路谐波及无功电流的一种 检测方法 $[\mathrm{J}]$. 电工技术学报, 1996, 11(3)：42-46。

[7] 李乔, 吴 捷. 自适应谐波电流检测方法用于有源电力滤波 器的仿真研究 [J]. 电工技术学报, 2004, 19 (12)：86-90。

[8] 徐在德, 范瑞祥, 荣彩霞, 等. 并联有源电力滤波器两种谐 波检测方法的研究 [J]. 电气传动, 2014, 44 (2) : 49-54。

[9] 谷金金, 王慧敏, 耿强, 等. 并联型有源电力滤波器的内模PI 控制 $[J]$. 电气传动, 2014，44（2）：68-74。

[10] 王亚芳, 秦岭, 王瑞祥. 级联型有源电力滤波器控制技术研 究 $[J]$. 电气传动, 2015.45(3) :34-36。

[11] 蔡鸿, 叶满园, 李宋. 基于DSP在线实线特定谐波消除技术 的两种方法 $[J]$. 电力自动化设备，2015.20（2）：15-20。

[12] 李达义, 孙玉鸿, 熊博, 等. 一种并联型有源电力滤波器的 新 型 控 制 方 法 $[\mathrm{J}]$. 电力系统 自 动 化, 2015, 38 (15) : 112-123。 
[13] 丁勇，张方华，王慧贞．基于特定消谐技术的四桥臂三相 逆变器 $[J]$. 电力电子技术, $2006,40(4)$ : 1-3。

[14] 王广柱. 并联型有源电力滤波器电流控制的等效原理 [J]. 中国电机工程学报，2006，26（15）：40-45。

[15] 王建学, 王锡凡, 张显, 等. 电力市场和过度期电力系统可中 断负荷管理 (二)--可中断负荷运营 $[\mathrm{J}]$. 电力自动化设备, 2004, 24 (6) : 1-5。
[16] 张兴, 张崇巍. PWM整流器及其控制 [M]. 北京: 机械出版社, 2012: 56-57。

[17] Dounda H. Overview of California ISO summer 2000 demand response programs $[\mathrm{A}]$. In:Proceedings of Power Engineering Society Winter Meeting[C]. Ohio (USA) : 2001. 228-223. 